\title{
The New Face of Hyperlipidemia Management: Proprotein Convertase Subtilisin/Kexin Inhibitors (PCSK-9) and Their Emergent Role As An Alternative To Statin Therapy
}

\author{
Lillian L. Smith; Juan F. Mosley II, Jarah Yates, Luke Caswell. \\ College of Pharmacy and Pharmaceutical Sciences, Florida A\&M University, Crestview, Florida, USA.
}

Received, November 16, 2015; Revised, January 29, 2016; Accepted, March 3, 2016; Published, March 3, 2016.

\begin{abstract}
This review analyzes Proprotein Convertase Subtilisin/Kexin 9 inhibitors (PCSK-9), a new medication class that has arisen in the last year to combat hypercholesterolemia. They are targeted towards patients who are unable to achieve acceptable low density lipoprotein (LDL) levels despite maximum statin therapy, as well as those who are unable to tolerate maximum statin therapy due to side effects such as myopathy or myalgia. Two of these medications have been released in the last year: alirocumab (Praluent) and evolocumab (Repatha). This article will overview this medication class, describe their pathophysiology, and analyze the clinical data from the numerous studies and trials done on both of these medications for their efficacy and safety outcomes. Data compiled on this new class of medications support the research that PCSK-9 inhibitors are both a safe and effective means of lowering the LDL levels of resistant or otherwise currently unmanaged hypercholesterolemia patients.
\end{abstract}

This article is open to POST-PUBLICATION REVIEW. Registered readers (see "For Readers") may comment by clicking on ABSTRACT on the issue's contents page.

\section{INTRODUCTION}

In today's society, unhealthy habits are commonplace amongst individuals. Subpar dietary intake and inadequate physical activity are amongst the most prevalent of these habits. Inevitably, these issues may cause an increase in cholesterol levels, specifically low-density lipoproteins (LDL), predisposing the individual to a plethora of medical and health issues. Diet, regular physical activity and smoking cessation are essential aspects in the treatment and reduction of LDL levels.(1) However, in some instances, these lifestyle changes are not drastic enough to control an individual's cholesterol levels. In these cases, physicians must initiate pharmacological therapy in order to combat unwanted cholesterol levels.

According to the most recent cholesterol guidelines, generally known as Adult Treatment Panel IV (ATP IV), a specific desired LDL goal is no longer recognized during the course of hyperlipidemic treatment. Alternatively, the guidelines suggest pharmacological therapy in certain population groups which are at an increased risk for atherosclerotic cardiovascular disease (ASCVD). These groups include patients with clinical atherosclerotic cardiovascular disease, LDL levels $\geq 190 \mathrm{mg} / \mathrm{dL}$ without clinical ASCVD, diabetic patients without clinical ASCVD who are between the ages of 40 and 75, with LDL levels between 70 and $189 \mathrm{mg} / \mathrm{dL}$, and non-diabetic patients without clinical ASCVD, who are between the ages of 40 to 75 with LDL levels between 70 and $189 \mathrm{mg} / \mathrm{dL}$.(2) High LDL levels affect a sizable amount of the US population, roughly 73.5 million (31.7\%) Americans as of this year.(3) These alarmingly high numbers in the adult population indicate a need for specific medical treatments designed for LDL level reduction.

Until recently, the medication classes used to treat high cholesterol included statins, selective cholesterol absorption inhibitors, bile acid sequestrants, fibrates, nicotinic acid, omega-3 fatty acids, and marine-derived omega-3 polyunsaturated fatty acids.(4) Of these medications, statin therapy is currently considered the mainstay of pharmacological treatment in hypercholesterolemia. The first statin to reach the market was lovastatin, which was FDA-approved in September of 1982.

Corresponding author: Juan F. Mosley II, Florida A\&M University, College of Pharmacy and Pharmaceutical Sciences, 153 W. Woodruff Avenue, Crestview, Fl. Juan.mosley@famu.edu 
Since its commercialization, six new statins have been approved, including two natural statins (simvastatin and pravastatin) and four synthetic statins (fluvastatin, atorvastatin, rosuvastatin, and pitavastatin).(5) According to the 2013 American College of Cardiology/American Heart Association (ACC/AHA) Guideline on the Treatment of Blood Cholesterol to Reduce Atherosclerotic Cardiovascular Risk in Adults, extensive evidence supports the use of statins for the prevention of ASCVD in many higher risk primary and all secondary prevention individuals without NYHA class II-IV heart failure and who were not receiving hemodialysis."(2) These guidelines recommend, in cases where statin therapy is ineffective, contraindicated, or intolerable due to side effects, that non-statin cholesterol lowering therapies such as those listed above may be initiated as either monotherapy or adjunctive therapy.(2) However, randomized clinical trials are needed to determine whether submaximal statin doses in combination with non-statin therapies, reduce ASCVD risk in statin-intolerant patients.(2) Such issues indicate that alternative medications would provide niche therapeutic options for statin-intolerant patients, as well as patients who are not treated effectively on statin therapy.

Proprotein convertase subtilisin/kexin type 9 inhibitors, also known as PCSK-9 inhibitors, are recently FDA approved therapies designed to dramatically lower the amount of cholesterol in the bloodstream.(6) This new class of cholesterol lowering medications may potentially cover the gaps in therapy neglected by both statin and other nonstatin therapies, as well as replace them in certain populations. PCSK-9 inhibitors may alter current cholesterol therapy and management due to the perceived effectiveness in clinical trials and benefits over current therapeutic options in certain populations.

On July 24, 2015, alirocumab (Praluent) (Sanofi, Bridgewater, NJ, USA) was the first FDA approved PCSK-9 inhibitor. Alirocumab's FDA-approved indications as of today includes adult patients with heterozygous familial hypercholesterolemia or patients with clinical atherosclerotic cardiovascular disease who require additional assistance lowering LDL cholesterol, in addition to diet and a maximally tolerated statin dose.(7) Alirocumab's notoriety as the only PCSK-9 inhibitor to be FDA approved was short-lived however, as the FDA approved evolocumab (Repatha) on August 27, 2015 as the second medication within the PSCK-9 inhibitor class. Repatha is approved by the FDA for the use in adult patients in combination to diet and maximallytolerated statin therapy for the treatment of heterozygous familial hypercholesterolemia, homozygous familial hypercholesterolemia, or clinical atherosclerotic cardiovascular disease.(8)

This review article offers a new and in depth look at this new class of medications, enabling prescribers, practitioners, and patients alike the information necessary to make sound clinical judgements. Each of the landmark clinical trials regarding alirocumab and evolocumab are detailed extensively, documenting their numerous successes in the realm of LDL lowering potential. Also, since the release of both of these new agents, analysis of these medications has been performed on long term risk reduction and cost-effectiveness. This will provide practitioners with enough evidence regarding these medications to choose the most effective LDL-lowering regimen for their patients.

\section{PCSK-9 INHIBITORS}

Despite attempts at adequate pharmacotherapy, low LDL-C levels cannot be achieved in certain patients and circumstances due to statin intolerance or ineffectiveness.(9) These current gaps in patient coverage provide ample opportunity for additional therapies to improve treatment. PCSK-9 inhibitors are quickly rising to the occasion, providing benefit to those neglected patients in clinical trials. Approximately twenty different medications are currently in development within this class, with two that are currently on the market.

The PCSK-9 inhibitors are monoclonal antibodies used to target the PCSK-9 gene, which as of now, the two FDA-approved formulations are administered subcutaneously, however intravenous formulations may also be utilized. At this juncture, the only information pertaining to the side effect profile of these medications is available through the approved PCSK-9 inhibitors. Though clinical trials are currently underway to determine the long-term effects of these medications, they appear to be relatively well tolerated. As of today, alirocumab and evolocumab have relatively the same documented side effects, including itching, swelling, pain, bruising at the injection site, nasopharyngitis, flu, and hypersensitivity reactions.(10) Drug-drug interactions are not expected to prove problematic due to target specificity, and alterations concerning the P450 enzymes, transport proteins, and QT 
interval remains largely unaffected. Monoclonal antibodies may cause infusion/injection site reactions, hypersensitivity reactions and localized toxicity pertaining to the area where the injection is administered.(11)

As apparent by the current FDA-approved formulations, the initial indication labeling for these medications are targeted towards patients with familial hypercholesterolemia.(12) Nevertheless, numerous clinical trials have been conducted on the basis that PCSK-9 inhibitors can serve as an alternative medication to those with statin intolerance or inadequate response to current therapies. Current pricing estimates for the PCSK-9 inhibitors, excluding alirocumab, range from approximately $\$ 7,000-\$ 12,000$ a year.(12) The newly approved agent, alirocumab, costs approximately $\$ 560$ a dose, which is equivalent to $\$ 40$ a day or $\$ 14,600$ per year.(13) Comparably, generic and brand name statins may cost $\$ 12$ per month (\$144 annually) with the latter costing \$500 per month $(\$ 6,000$ annually).(14) Assuming a median annual cost of $\$ 10,000$ per year for its likely indication of familial hypercholesterolemia, approximately $\$ 16$ billion in cost can be expected. With the addition of statin intolerance as an indication, a supplemental $\$ 20$ billion can be expected.(12)

Since marketing of these products have begun, they have been regarded as some of the most effective LDL lowering therapies to date. However, this has not given them cause to be added to regular regimens or formularies. In fact, the U.K.'s National Institute for Health and Care Excellence (NICE) has decided not to add evolocumab to the national formulary due to expense issues and the fact that no evidence exists that confirms evolocumab's usefullness in improving long-term clinical outcomes.(15) Additionally, the New England Comparative Effectiveness Public Advisory Council conducted a cost-effectiveness study on this class. Research findings suggest that a cost reduction of 60$63 \%$ is necessary for this class to become cost effective, despite their considerable reductions in regards to LDL levels.(15) In the United States, these agents have made some headway by allowing coupons and deals to be utilized by patients, in order to make these drugs much more affordable. Furthermore, deals are being struck between Amgen and various insurance companies to create a 'pay-byperformance' deal with evolocumab, in order to reduce prices further for patients.(16)

\section{PATHOPHYSIOLOGY}

Abifadel and co-investigators first discovered the breakthrough of the PCSK-9 gene during research of two French families with clinical familial hypercholesterolemia. During initial research, the families' phenotypes possessing gain-of-function mutations were discovered, however, researchers at the time were unable to identify correlations connecting the mutations with familial hypercholesterolemia. Analysis derived from the research did indicate mutations in the PCSK-9-a gene, however, a direct correlation at the time was unknown in relation to metabolism of cholesterol.(17)

Endogenously, PCSK-9 is principally synthesized in the small intestines and liver as a neural apoptosis regulated convertase-1 (NARC-1). Following cleavage of the prodomain via intracellular autocatalytic conversion, the PCSK-9 is secreted from liver cells acting as a serine protease.(17,18) Following secretion from the liver, PCSK-9 binds to LDL receptors, which directly influence plasma LDL concentrations. Properly regulated PCSK-9 induces the lysosomal degradation of the LDL receptor following the initial binding. The degradation of the LDL receptor prevents the reutilization of the receptor to the hepatocyte surface, which inhibits the catabolism of circulating plasma LDL.(18)

In the case of hypercholesterolemia, gain-offunction mutations in the PCSK-9 gene alter natural endogenous function, particularly selected missense mutations. In this case, an abnormal alteration leads to hyperactivity of the PCSK-9 gene, or the addition of an entirely new function.(19) Consequently, the overactive PCSK-9 gene degrades LDL receptors in excess, reducing LDL removal from the blood, thus increasing LDL levels.(20)

Studies indicate that the LDL receptor does not undergo lysosomal degradation in cases where PCSK-9 protease is no longer present or in cases of deficiency. Conversely, in cases involving the absence of PCSK-9 protease, the receptors are transported back to the plasma membrane after binding with plasma cholesterol, which will undergo lysosomal degradation. Thus, PCSK-9 prevents this transport of the receptors allowing the liver to decrease the circulating plasma LDL.(17) Therefore, PCSK-9 inhibition could be expected to reduce LDL levels in patients by allowing more LDL receptors to catabolize the lipoprotein. These findings support the induction of PCSK-9 inhibitors as a viable class of 
medications to reduce LDL levels in patients with familial or severe hypercholesterolemia.

\section{Clinical Trials}

PCSK-9 inhibitors have undergone several Phase II and Phase III trials. Evolocumab and alirocumab are two PCSK-9 inhibitors that have currently undergone the most research and appear to be approaching approval by the FDA. Evolocumab has been evaluated in both short and long term studies to determine both the product effectiveness, as well the proposed safety profile. Some of the more notable studies conducted include the GAUSS-2 trial, the OSLER trial, and the RUTHERFORD trial. Clinical trials involving alirocumab include ODYSSEY COMBO II, ODYSSEY FH, and other monotherapeutic Phase III trials, which evaluated therapeutic efficacy and safety profile. Based on evidence derived from these clinical trials, Evolocumab and alirocumab would initially be indicated for patients who are intolerant to statins and/or experiencing familial hypercholesterolemia.

\section{EVOLOCUMAB}

\section{GAUSS-2}

The GAUSS-2 (Goal Achievement after Utilizing an Anti-PCSK-9 Antibody in Statin Intolerant Subjects) Randomized, Placebo-Controlled Phase III Clinical Trial compared subcutaneous Evolocumab vs. oral Ezetimibe in hypercholesterolemia patients which were unable to tolerate statin therapy.(21) Ezetimibe was initially chosen as the comparison medication, largely due to a favorable tolerability profile commonly used in statin-intolerant patients. Patients received a randomized 2:2:1:1 distribution of therapies, including subcutaneous evolocumab 140 mg every two weeks (Q2W) or evolocumab $420 \mathrm{mg}$ once monthly $(\mathrm{QM})$. Both trial therapies were dosed with daily oral placebo or subcutaneous placebo Q2W or QM both with daily oral ezetimibe.

Primary endpoints included percent change from baseline in LDL levels at the mean of weeks 10 to 12 and again at week 12, whereas the secondary endpoints encompassed results such as change from baseline in LDL, percent of patients with LDL $<70$ $\mathrm{mg} / \mathrm{dl}$, percent change from baseline in non-highdensity lipoprotein cholesterol (HDL), apolipoprotein B, total cholesterol/HDL-C ratio, apolipoprotein $\mathrm{B} /$ apolipoprotein $\mathrm{A}-\mathrm{I}$ ratio, lipoprotein(a), triglycerides, HDL-C, and very lowdensity lipoprotein (VLDL).(21)
Of the initial 307 patients originally participating in the study, a large portion, approximately $94 \%$ (290 patients), completed the trial. Amongst the primary endpoints, evolocumab produced significant reductions in LDL levels at weeks 10 to 12, as well as week 12. Furthermore, evolocumab yielded a $56.1 \%$ decrease $(95 \%$ confidence interval [CI]: $59.7 \%$ to $52.5 \%$ ) in LDL levels from baseline with the $140 \mathrm{mg}$ Q2W dose, and a $55.3 \%$ decrease $(95 \%$ CI: $58.3 \%$ to $52.3 \%$ ) with the 420 gm QM dose, in comparison to Ezetimibe QD with placebo Q2W, which produced a $36.9 \%$ decrease (95\% CI: $42.3 \%$ to $31.6 \%$ ) and Ezetimibe QD with placebo QM, which yielded a $38.7 \%$ decrease (95\% CI: $43.1 \%$ to $34.3 \%$ ) in LDL from baseline at weeks 10 to 12 (p $<0.001)$.(19) The mean percent reductions from baseline to week 12 were very similar, as well $(\mathrm{p}<$ 0.001). Similarly, GAUSS-2 indicated that evolocumab-treated patients were more likely to achieve LDL-C target levels than ezetimibe-treated patients.

Evolocumab demonstrated clear superiority to ezetimibe in returning patients to target LDL levels, both at week 12 and at the means of weeks 10 and 12 in patients in all LDL risk categories. The results of the secondary endpoints illustrated that, when compared with ezetimibe, evolocumab led to significant reductions in apolipoprotein $B$, lipoprotein(a), non-HDL-C, and the apolipoprotein B/apolipoprotein A-I and total cholesterol/HDL-C ratios. Safety outcomes of evolocumab identified myalgia as a significant side effect throughout the course of therapy, occurring in $8 \%$ of patients. However, the probability of patients experiencing myalgia occurred primarily in those taking a lowdose statin alongside evolocumab (statin vs. nostatin: $17 \%$ vs. $6 \%$ ). Additionally, the trial detected no binding or neutralizing antibodies in the evolocumab treated patients.(21)

Final data compilation proved that evolocumab produced a significant reduction in LDL levels in patients intolerant to statins. In high risk patients, more than $75 \%$ of patients achieved LDL levels of less than $100 \mathrm{mg} / \mathrm{dL}$ when treated with evolocumab, as compared to less than $10 \%$ of patients who were treated with ezetimibe. Evolocumab proved tolerable in approximately $96 \%$ of patients upon completion of the trial. Furthermore, therapeutic benefits of evolocumab, when placed alongside current ACC/AHA guidelines, would provide a promising alternative therapy for statin-intolerant patients with markedly elevated LDL levels. Concurrently, the 
clean safety profile of evolocumab may allow its integration into cholesterol management, replacing current alternative agents such as ezetimibe.(21)

\section{OSLER}

The OSLER trial, or the Efficacy and Safety of Longer-Term Administration of Evolocumab (AMG 145) in patients with hypercholesterolemia: 52-week wesults from the open-label study of long-term evaluation against LDL-C (OSLER) Randomized Trial evaluated the long term effectiveness of evolocumab vs. the standard of care in lowering LDL levels. OSLER was a global study, conducted at 156 centers involving at least 1 of the 4 Phase 2 clinical trials on the PCSK-9 inhibitors: the MENDEL trial, the GAUSS trial, the RUTHERFORD trial, and the LAPLACE-TIMI 57 trial.(22) Approximately 81\% (1104) of patients from the Phase 2 parent trials participated in the OSLER clinical trial, which allowed potential evaluation of the long term effects of the medication. Regardless of the therapy patients received during the previous Phase 2 trials, the participants were randomized in a 2:1 fashion to one of the two treatment groups, either evolocumab 420 mg SC every 4 weeks plus standard of care (SOC) (736 patients) or SOC alone (368 patients). Notably, 691 patients $(62.6 \%)$ were on statin therapy at baseline, with $64.9 \%$ of those patients in the evolocumab plus SOC group and $57.9 \%$ in the SOC group.

Two primary endpoints were examined: one evaluating the proposed safety profile, and the other determining efficacy. The primary efficacy endpoint aimed to characterize the effects of longer-term administration of evolocumab as assessed by LDLC, non-high-density lipoprotein cholesterol (nonHDL-C), apolipoprotein (Apo) B, ratio of total cholesterol to HDL-C, and ApoB/ApoA1 ratio in patients with hypercholesterolemia. The primary safety endpoints included the incidence of adverse events (AEs), serious AEs, AEs leading to product discontinuation, the incidence of creatine kinase and liver function test abnormalities and the incidence and percentage of patients who developed antiEvolocumab antibodies (binding or neutralizing).(22)

Results summarizing the efficacy endpoints signified that patients not taking evolocumab in the Phase 2 parent studies exhibited large initial LDL-C reductions determined at 12 weeks after starting evolocumab treatment in OSLER (51.8\% [SE, 1.6\%] reduction from the parent study baseline; $P<0.0001$ versus baseline), with reductions maintained for the entire 52 weeks $(52.3 \%$ [SE, $1.8 \%$ ] at week 52; $P<0.0001$ versus baseline). Moreover, patients receiving one of six dosing regimens of evolocumab $(70,105,140 \mathrm{mg}$ every 2 weeks or $280,350,420 \mathrm{mg}$ every 4 weeks) in the parent studies and received continued evolocumab treatment in OSLER had persistent reductions in LDL-C when compared with baseline (from $50.4 \%$ [SE, $0.8 \%$ ] at the end of the parent study to $52.1 \%$ [SE, $1.0 \%$ at 52 weeks paired $t$-test, $P=0.31) .{ }^{20}$ Participants which discontinued evolocumab due to random assignment to SOC in OSLER experienced quickly rising LDL levels. LDL-C reduction in these patients decreased from $53.1 \%$ [SE, 1.2\%] at the start of OSLER to just $17.9 \%$ [SE, 1.2\%] after 4 weeks of treatment with SOC alone. Furthermore, LDL levels in these patients then continued to rise to near baseline levels (5.8\%) [SE, 1.2\%] within 12 weeks without a rebound effect. Additionally, increases in HDL and ApoA1 levels were observed in patients who continued the evolocumab. Median triglyceride levels experienced a moderate decrease in the evolocumab plus SOC group over the 52 week duration.(22)

Adverse events occurred in 868 patients (78.6\%), 269 patients (73.1\%) in the SOC group and 599 patients $(81.4 \%)$ in the evolocumab plus SOC group. Most commonly, nasopharyngitis, upper respiratory tract infections, influenza, arthralgia, and back pain were reported, though only $5.6 \%$ of all AEs were considered related to evolocumab treatment. Of the 23 serious adverse effects noted, none were determined relatable to the evolcumab treatment arm. Moreover, no neutralizing or binding antibodies to evolocumab were detected throughout the trial. Participants assigned Evolocumab plus SOC, 98 patients achieved LDL levels of $<25$ $\mathrm{mg} / \mathrm{dL}, 409$ patients achieved levels of $<50 \mathrm{mg} / \mathrm{dL}$, and 323 patients remained at levels of $\geq 50 \mathrm{mg} / \mathrm{dL}$. Though low LDL levels in these patients were of concern, overall or serious AEs, elevations in creatine kinase and aminotransferases were not appreciably greater. However, headaches, dizziness, insomnia, and back pain were more predominant in these patients. One patient in the evolocumab plus SOC group died during the study, however, the patient had a history of significant coronary artery disease, and a ventricular aneurysm was found during month 5 of OSLER.(22)

In conclusion, evolocumab plus SOC proved more effective than SOC alone in the treatment of 
hypercholesterolemia. Significant reductions in LDL levels were observed for the entire 52 week duration of the trial. Furthermore, patients previously treated with evolocumab showed persistent reductions in their LDL levels throughout the 52 week OSLER trial when they continued evolocumab. More studies to evaluate longer term safety profiles and therapeutic efficacy are warranted for evolocumab.

\section{RUTHERFORD}

The RUTHERFORD (Reduction of LDL-C With PCSK-9 Inhibition in Heterozygous Familial Hypercholesterolemia Disorder) trial was a phase II, multicentered, double-blind, placebo-controlled trial conducted in over 20 lipid clinics around the world. The primary objective compared 12 weeks of subcutaneously administered AMG-145 (evolocumab) $350 \mathrm{mg}$ or $420 \mathrm{mg}$ given every four weeks alongside a placebo group. After initial screening, 56 participants received the AMG-145 $350 \mathrm{mg}$ dose, while 56 participants were given the AMG-145 $420 \mathrm{mg}$ dose. Lastly, of the 168 randomized participants, 56 were allocated to the placebo in a 1:1:1 ratio. The primary outcome monitored changes in LDL-C from the baseline in participants, all of which experienced heterozygous familial hypercholesterolemia. Alternatively, the secondary outcome objectives included assessments in the absolute change in LDL-C, percentage change in non-high density lipoprotein cholesterol, ApoB, total cholesterol/HDL-C ratio and ApoB/apolipoprotein A1 ratio as well as the safety and tolerability. Inclusion criteria for this trial included men and women aged 18 to 75 years old with clinically diagnosed heterozygous familial hypercholesterolemia, with LDL levels over 100 $\mathrm{mg} / \mathrm{dL}$ and triglycerides less than $400 \mathrm{mg} / \mathrm{dL}$ despite at least 4 weeks of stable statin and other lipid lowering therapies before screening. Exclusion criteria included patients with "homozygous familial hypercholesterolemia; patients who had LDL or plasma apheresis within 12 months of randomization; heart failure of New York Heart Failure Association class III or IV or left ventricular ejection fraction $<30 \%$; any acute or unstable cardiac event with planned intervention within 3 months of randomization; type 1 diabetes mellitus or newly diagnosed or poorly controlled (hemoglobin A1c $>8.5 \%$ ) type 2 diabetes mellitus; systolic blood pressure $>160 \mathrm{mmHg}$ or diastolic blood pressure $>100 \mathrm{mmHg}$; thyroid-stimulating hormone $<1 \times$ lower limit of normal or $>1.5 \times$ upper limit of normal
(ULN); estimated glomerular filtration rate $<30$ $\mathrm{mL} / \mathrm{min} / 1.73 \quad \mathrm{~m}^{2}$; persistent aspartate aminotransferase or alanine aminotransferase $>2 \times$ ULN or creatine kinase $>3 \times$ ULN." (23)

Results of the study indicated that both arms including AMG-145 doses were more effective in reducing LDL-C levels than placebo. AMG-145 350 $\mathrm{mg}$ and $420 \mathrm{mg}$ showed a percentage reduction of LDL-C levels of $43 \%$ and $55 \%$ respectively, as compared with placebo, which only reduced LDL levels in $3 \%$ of patients $(\mathrm{P}<0.001$ for both dose groups). At 12 weeks, $70 \%$ and $89 \%$ of individuals receiving AMG-145 $350 \mathrm{mg}$ and $420 \mathrm{mg}$ experienced LDL-C reductions to levels of less than $100 \mathrm{mg} / \mathrm{dL}$, and $44 \%$ and $65 \%$ of these patients achieved levels of less than $70 \mathrm{mg} / \mathrm{dL}$, respectively.(23) Furthermore, $95 \%$ of patients in the AMG-145 treatment arms experienced LDL-C level reductions of at least $15 \%$. More impressively, $52 \%$ of these patients achieved 50\% LDL-C level reductions or more. There was a significant dosedependent reduction in lipoprotein (a) with AMG 145 therapy of $23 \%$ and $32 \%$ compared with placebo. Reductions in total cholesterol, ApoB, nonHDL-C and triglycerides showed modest reductions in the AMG-145 arms as compared with placebo. (23)

Treatment in both AMG-145 treatment arms did not result in any clinically significant safety problems. However, there was a higher incidence of treatment-related adverse events in the AMG-145 $420 \mathrm{mg}$ arm, yet no evidence existed to correlate these adverse events with the dose itself. The AMG145 treatment arms were associated with the following common side effects: injection site pain (3.6\% - $420 \mathrm{mg}$ dose, $7.3 \%$ - $350 \mathrm{mg}$ dose, $1.8 \%$ placebo), and headache (1.8\% - $420 \mathrm{mg}$ dose, $5.5 \%$ $350 \mathrm{mg}$ dose, $0.0 \%$ - placebo), and skin burning sensation $(3.6 \%$ - $420 \mathrm{mg}$ dose, $1.8 \%$ - $350 \mathrm{mg}$ dose, $0.0 \%$ - placebo).(23)

Overall, AMG-145, otherwise known as evolocumab, showed an efficacious reduction in LDL-C levels in both treatment groups as compared to placebo. This study concludes that with the use of concomitant statin therapy, with or without ezetimibe, AMG-145 may be an effective means of assisting in the reduction of high LDL-C levels in patients with heterozygous familial hypercholesterolemia. 


\section{ALIROCUMAB}

\section{ODYSSEY COMBO II}

This trial, referred to as COMBO II, compared alirocumab to ezetimibe as adjunctive therapy to a maximally tolerated statin therapy in high cardiovascular risk patients with inadequately controlled hypercholesterolemia.(24) COMBO II was conducted as a double-blind, double-dummy, active-controlled, parallel-group, 104-week study conducted across 126 in Europe, Israel, North America, South Africa and South Korea, in which patients were randomized in a 2:1 fashion to receive $75 \mathrm{mg}$ biweekly doses of subcutaneous alirocumab plus oral placebo or oral ezetimibe plus subcutaneous placebo, both on a background of statin therapy. 720 participants were eligible to participate in this study. 479 and 241 patients were allocated to the alirocumab and ezetimibe groups, respectively.

The primary endpoint of this trial was percent change in calculated LDL-C from baseline to Week 24. Safety outcomes were measured through laboratory data that was collected and analyzed from the beginning of the study to the end, as well as monitoring adverse reaction reports.

The mean \pm SD baseline calculated LDL-C concentration was $2.8 \pm 0.9 \mathrm{mmol} / \mathrm{L} ; 66.7 \%(\mathrm{n}=$ 480) were taking atorvastatin $40 / 80 \mathrm{mg} /$ day or rosuvastatin $20 / 40 \mathrm{mg} / \mathrm{day}$, and $2.1 \%(\mathrm{n}=15)$ were on simvastatin $80 \mathrm{mg}$. For the primary endpoint, mean \pm standard error (SE) reductions in LDL-C from baseline to Week 24 were $-50.6 \pm 1.4 \%$ in the alirocumab arm and $-20.7 \pm 1.9 \%$ in the ezetimibe arm, both on a background of maximally tolerated statin therapy, with a statistically significant difference of the means \pm SE between groups of $-29.8(95 \%$ CI -34.4 to $-25.3, \mathrm{P}<0.0001)$. The proportion of patients who achieved the target LDL$\mathrm{C}$ of $<1.8 \mathrm{mmol} / \mathrm{L}$ at Week 24 (ITT analysis) was $77.0 \%$ in the alirocumab arm and $45.6 \%$ in the ezetimibe arm $(\mathrm{P}<0.0001)$. The overall percentages of patients who experienced at least one TEAE were $71.2 \%$ in the alirocumab arm and $67.2 \%$ in the ezetimibe arm. A higher proportion of patients in the alirocumab group experienced TEAEs leading to treatment discontinuation ( 7.5 vs. $5.4 \%$ ), with no specific pattern in type of adverse event.(24)

In conclusion, alirocumab was proven to be effective in lowering LDL-C from baseline, more than the current standard of therapy, ezetimibe. Alirocumab was also well-tolerated in patients, providing a clean profile similar to that of ezetimibe.(24)

\section{MONOTHERAPY WiTh THE PCSK-9 INHIBITOR ALIROCUMAB}

This Phase III, randomized, double-blind, doubledummy study evaluated the efficacy and safety of alirocumab when compared with ezetimibe in hypercholesterolemic patients at moderate cardiovascular risk not receiving statins or other lipid-lowering therapy.(25) Patients received either $10 \mathrm{mg} /$ day of ezetimibe or $75 \mathrm{mg}$ every 2 weeks of subcutaneous alirocumab with dose titrated to 150 mg Q2W at week 12 if week 8 LDL-C was $\geq 70$ $\mathrm{mg} / \mathrm{dL}$. The primary endpoint of this trial was the mean LDL percent change from baseline to 24 weeks in alirocumab as compared to ezetimibe. The safety endpoints were assessed throughout the study by adverse event reporting, injection site reactions, laboratory data, vital signs, physical signs, and electrocardiogram. Also, treatment-emergent adverse events (TEAEs) were assessed, which were defined as any adverse events that emerged, worsened, or became serious during the course of treatment, regardless of whether they were deemed drug-related. The TEAE period, as defined by the research team, included the period from the first dose to 70 days (10 weeks) after the last injection.(25)

In total, 103 patients participated and were further randomized into two control groups. LDL-C reductions in the on-treatment analysis at week 12 were 53 (2)\% with alirocumab versus 20 (2)\% with ezetimibe. Alirocumab produced greater reductions in apolipoprotein B, total cholesterol, and non-high density lipoprotein cholesterol as compared to ezetimibe at weeks 24. Moderate reductions in ApoA and triglycerides were recorded, while increases in high-density lipoprotein cholesterol were observed in both treatment groups. There were no significant differences between alirocumab and ezetimibe arms in these cases.(25) Overall, 69\% of patients in the alirocumab group experienced TEAEs, as compared to $78 \%$ in the ezetimibe group. One patient in each group experienced a serious TEAE, but both patients recovered and completed the study.

This was the first Phase III study to evaluate the $75 \mathrm{mg}$ twice weekly dose of alirocumab. Overall, results suggested that this dose of alirocumab was superior to ezetimibe over a 24 week period. In moderate cardiovascular risk patients, $75 \mathrm{mg}$ subcutaneous, Q2W alirocumab was effective in reducing LDL levels over $50 \%$ in most patients, 
which is comparable to the efficacy of high intensity statins. Dose titration in the alirocumab group occurred at week 8 in patients who had a LDL of 70 $\mathrm{mg} / \mathrm{dL}$ or under, though it was intended to occur in patients with an LDL of under $100 \mathrm{mg} / \mathrm{dL}$ or over. This titration, however, was postulated to have had a negligible effect if it had occurred at the higher level. Alirocumab showed tolerability and safety comparable to that of ezetimibe. This is noteworthy due to the fact that ezetimibe is the preferred agent in patients who have statin intolerance due to its favorable profile.(25)

\section{ODYSSEY FH STUDIES}

The ODYSSEY studies are a compilation of three clinical trials (FH I, FH II, and HIGH FH) conducted to study the effect of alirocumab on familial hypercholesterolemia $(\mathrm{FH})$. These studies are a multicenter, multinational, randomized, doubleblind, placebo-controlled in over 800 patients who are not adequately controlled with a maximallytolerated stable daily dose of statin for $\geq 4$ weeks prior to the screening visit, with or without other lipid-lowering therapy.(26)

Patients were randomized 2:1, receiving 1 vial of either subcutaneous alirocumab or placebo Q2W for 78 weeks. In FH I and FH II, if patients had a week 8 LDL-C level of $\geq 70 \mathrm{mg} / \mathrm{dL}$, patients received a dose titration from 75 to $150 \mathrm{mg}$ alirocumab Q2W at week 12. In HIGH FH, patients received a dose of $150 \mathrm{mg}$ Q2W throughout the entire study. The primary efficacy endpoint in all three studies is the percent change in calculated LDL-C from baseline to Week 24.

These studies are currently ongoing, and will assess the efficacy of alirocumab in familial hypercholesterolemia, as well as the long term efficacy. Patients will also be allowed to participate in an open-label extension study at the end of the double-blind treatment period, which will allow for further assessment of the efficacy and safety of alirocumab.(26)

\section{CONCLUSION}

Hypercholesterolemia affects millions of individuals, many of whom seek treatment through conventional therapy, namely statins. While statins have been the mainstay of cholesterol therapy for decades, there are still a disproportionately high number of patients who fall between the cracks, not receiving adequate cholesterol management. These gaps in therapeutic management leave room for emerging medications, such as the PCSK-9 inhibitors to fill in the gaps.

The emergence of a newly approved PCSK-9 inhibitors, with additional medications sure to follow, will provide new methods in the management of hypercholesterolemia in the near future. Furthermore, individuals with familial hypercholesterolemia would receive specialized therapy, due to the targeting of FH in the PCSK-9 inhibitor clinical trials. PCSK-9 inhibitors may prove to be a revolutionary new way to treat hypercholesterolemia adjunctively with statins, as well as in cases of statin intolerance. Most clinical trials for these medications are currently in Phase III, though alirocumab and evolocumab have recently been FDA-approved, meaning increased availability of other PCSK-9 inhibitors in cholesterol treatment could be on the horizon. PCSK-9 inhibitors could provide benefit to currently inadequately treated hypercholesterolemia patients, potentially closing the gaps of therapy.

\section{REFERENCES}

1. Heart.org. Lifestyle Changes and Cholesterol. 2015. Available at: http://www.heart.org/HEARTORG/Conditions/Chol esterol/PreventionTreatmentofHighCholesterol/Lifes tyle-Changes-and-

Cholesterol_UCM_305627_Article.jsp. Accessed July 3,2015

2. Stone N, Robinson J, Lichtenstein A et al. 2013 ACC/AHA Guideline on the Treatment of Blood Cholesterol to Reduce Atherosclerotic Cardiovascular Risk in Adults. Journal of the American College of Cardiology. 2014;63(25):28892934.

3. Heart Disease and Stroke Statistics - 2015 Update A Report From the American Heart Association. AHA Journals. 2015:e288-e290. Available at: http://circ.ahajournals.org/content/early/2014/12/18/ CIR.0000000000000152.full.pdf. Accessed July 2, 2015.

4. Heart.org. Drug Therapy for Cholesterol. 2015. Available

at: http://www.heart.org/HEARTORG/Conditions/Chol esterol/PreventionTreatmentofHighCholesterol/Drug -Therapy-for-

Cholesterol_UCM_305632_Article.jsp. Accessed July $3,201 \overline{5}$.

5. ENDO A. A historical perspective on the discovery of statins. BEPPU T, ed. Proceedings of the Japan Academy Series B, Physical and Biological Sciences. 2010;86(5):484-493. doi:10.2183/pjab.86.484. 
6. Gregory Curfman M. PCSK-9 inhibitors: a major advance in cholesterol-lowering drug therapy Harvard Health Blog. Harvard Health Blog. 2015. Available

at:

http://www.health.harvard.edu/blog/PCSK-9-

inhibitors-a-major-advance-in-cholesterol-loweringdrug-therapy-201503157801. Accessed July 2, 2015.

7. Prnewswire.com. Regeneron and Sanofi Announce FDA Approval of Praluent ${ }^{\circledR}$ (alirocumab) Injection, the First PCSK-9 Inhibitor in the U.S., for the Treatment of High LDL Cholesterol in Adult Patients. 2015. Available at: http://www.prnewswire.com/newsreleases/regeneron-and-sanofi-announce-fdaapproval-of-praluent-alirocumab-injection-the-firstPCSK-9-inhibitor-in-the-us-for-the-treatment-ofhigh-ldl-cholesterol-in-adult-patients300118572.html. Accessed August 30, 2015.

8. Fda.gov. FDA approves Repatha to treat certain patients with high cholesterol. 2015. Available at: http:/www.fda.gov/NewsEvents/Newsroom/PressA nnouncements/ucm460082.htm. Accessed August 30, 2015.

9. Cholesterol-busting PCSK-9 drugs. The Pharmaceutical Journal : A Royal Pharmaceutical Society publication. 2015;294(7858). Available at: http://www.pharmaceutical-journal.com/news-andanalysis/features/PCSK-9-inhibitors-the-nextcholesterol-lowering-blockbusters/20068181.article. Accessed July 3, 2015.

10. Fda.gov. FDA approves Praluent to treat certain patients with high cholesterol. 2015. Available at: http:/www.fda.gov/NewsEvents/Newsroom/PressA nnouncements/ucm455883.htm. Accessed August 20, 2015.

11. Joseph L, Robinson J. Proprotein Convertase Subtilisin/Kexin Type 9 (PCSK-9) Inhibition and the Future of Lipid Lowering Therapy. Progress in Cardiovascular Diseases. 2015;19(31):19-31. doi. 1016/j.pcad.2015.04.004.

12. Shrank W, Lotvin A, Singh S, Brennan T. In The Debate About Cost And Efficacy, PCSK-9 Inhibitors May Be The Biggest Challenge Yet. Healthaffairsorg. 2015. Available at: http://healthaffairs.org/blog/2015/02/17/in-thedebate-about-cost-and-efficacy-PCSK-9-inhibitorsmay-be-the-biggest-challenge-yet/. Accessed July 14, 2015.

13. Herper M. The FDA Approves A Potent And Pricey Cholesterol-Lowering Shot. Forbes. 2015. Available at:

http://www.forbes.com/sites/matthewherper/2015/07 /24/the-fda-approves-a-potent-and-pricey-

cholesterol-lowering-shot/. Accessed August 20, 2015.

14. Consumer Health Choices. Statins - Consumer Health Choices. 2015. Available at: http://consumerhealthchoices.org/wp-

content/uploads/2012/08/BBD-Statins-2pg.pdf.

Accessed July 15, 2015.

15. Medpagetoday.com,. "Update: PCSK-9 Inhibitors Hit The Market". N.p., 2016. Web. 27 Jan. 201

16. Weisman, Robert. "Harvard Pilgrim Strikes 'PayFor-Performance' Deal For Cholesterol Drug - The Boston Globe". BostonGlobe.com. N.p., 2015. Web. 27 Jan. 2016.

17. Verbeek R, Stoekenbroek RM, Hovingh GK. PCSK9 inhibitors: Novel therapeutic agents for the treatment of hypercholesterolemia. Eur J Pharmacol. 2015 Sep 15;763(Pt A):38-47. doi: 10.1016/j.ejphar.2015.03.099.

18. Santos RD, Watts GF. Familial hypercholesterolaemia: PCSK-9 inhibitors are coming. Lancet. 2015 Jan 24;385(9965):307-10. doi: 10.1016/S0140-6736(14)61702-5.

19. Horton J, Cohen J, Hobbs H. Molecular biology of PCSK-9: its role in LDL metabolism. Trends in Biochemical Sciences. 2007;32(2):71-77. doi:http://dx.doi.org/10.1016/j.tibs.2006.12.008.

20. Genetics Home Reference. PCSK-9 gene. 2015. Available at: http://ghr.nlm.nih.gov/gene/PCSK-9. Accessed July 17, 2015.

21. Stroes E, Colquhoun D, Sullivan D et al. Anti-PCSK9 Antibody Effectively Lowers Cholesterol in Patients With Statin Intolerance. Journal of the American College of Cardiology. 2014;63(23):25412548. doi.10.1016/j.jacc.2014.03.

22. Koren MJ, Giugliano RP, Raal FJ, Sullivan D, Bolognese M, Langslet G, Civeira F, Somaratne R, Nelson P, Liu T, Scott R, Wasserman SM, Sabatine MS; OSLER Investigators. Efficacy and safety of longer-term administration of evolocumab (AMG 145) in patients with hypercholesterolemia: 52-week results from the Open-Label Study of Long-Term Evaluation Against LDL-C (OSLER) randomized trial. Circulation. 2014 Jan 14;129(2):234-43. doi: 10.1161/CIRCULATIONAHA.113.007012.

23. Raal F, Scott R, Somaratne R, Bridges I, Li G, Wasserman SM, Stein EA. Low-density lipoprotein cholesterol-lowering effects of AMG 145, a monoclonal antibody to proprotein convertase subtilisin/kexin type 9 serine protease in patients with heterozygous familial hypercholesterolemia: the Reduction of LDL-C with PCSK-9 Inhibition in Heterozygous Familial Hypercholesterolemia Disorder (RUTHERFORD) randomized trial. Circulation. 2012 Nov 13;126(20):2408-17. doi: 10.1161/CIRCULATIONAHA.112.144055.

24. Cannon C, Cariou B, Blom D et al. Efficacy and safety of alirocumab in high cardiovascular risk patients with inadequately controlled hypercholesterolaemia on maximally tolerated doses 
of statins: the ODYSSEY COMBO II randomized controlled trial. European Heart Journal. 2015;36(19):1186-1194. doi: http://dx.doi.org/10.1093/eurheart/ehv028.

25. Roth EM, Taskinen MR, Ginsberg HN, Kastelein JJ, Colhoun HM, Robinson JG, Merlet L, Pordy R, Baccara-Dinet MT. Monotherapy with the PCSK-9 inhibitor alirocumab versus ezetimibe in patients with hypercholesterolemia: results of a 24 week, double- blind, randomized Phase 3 trial. Int J Cardiol. 2014 Sep;176(1):55-61. doi: 10.1016/j.ijcard.2014.06.049.

26. Kastelein JJ, Robinson JG, Farnier M, Krempf M, Langslet G, Lorenzato C, Gipe DA, Baccara-Dinet MT. Efficacy and safety of alirocumab in patients with heterozygous familial hypercholesterolemia not adequately controlled with current lipid-lowering therapy: design and rationale of the ODYSSEY FH studies. Cardiovasc Drugs Ther. 2014 Jun;28(3):2819. doi: 10.1007/s10557-014-6523-z. 